\title{
STABILITY OF TIME VARYING SYSTEMS
}

\author{
Fritz Colonius \\ Institut für Mathematik \\ Universität Augsburg \\ Augsburg, Germany \\ Wolfgang Kliemann \\ Department of Mathematics \\ lowa State University \\ Ames, lowa
}

\begin{abstract}
The stability behavior of time varying systems can be studied using the concept of Lyapunov exponents and their corresponding Lyapunov subspaces. For linear time varying systems the entire Lyapunov spectrum can be approximated by the Floquet exponents of periodic systems. This leads to a variety of stability results, including the characterization of stability radii. Furthermore, a structural stability type theorem shows that stability features of time varying hyperbolic systems persist under small perturbations. For nonlinear time varying systems a stable manifold theorem allows us to interpret the linear results for the nonlinear system locally around an equilibrium point.
\end{abstract}

\section{INTRODUCTION}

Two standard techniques for the stability analysis of dynamical systems were developed in M.A. Lyapunov's thesis from 1893: A Lyapunov function describes the convergence behavior of all trajectories in (at least) a neighborhood of the limit set under consideration, where the limit set could be e.g. a fixed point or a periodic orbit. On the other hand, Lyapunov exponents describe the exponential growth behavior of the trajectories depending on their initial values. This approach gives a detailed picture of the stable and unstable directions of the system at a limit set. Our aim is to present a stability theory for time varying systerns using the concept of Lyapunov exponents. Our hope is that this theory will lead to design principles for time varying mechanical systems and structures.

A brief summary of the Lyapunov exponents approach for time invariant systems $\dot{y}=X_{0}(y)$ looks like this: Let $y^{*}$ be a fixed point of the vector field $X_{0}$. Linearize the system around $y^{*}$, i.e. consider the linear system $\dot{x}=A x$, where the matrix $A=D_{y} X_{0}\left(y^{*}\right)$ is the Jacobian of $X_{0}$ at the equilibrium point $y^{*}$. Compute the eigenvalues and eigenspaces of $A$, and map the eigenspaces to the nonlinear state space as stable, unstable, and center manifolds. These invariant manifolds determine the phase portrait of $\dot{y}=X_{0}(y)$ around $y^{*}$ and, in particular, describe the stability behavior locally around $y^{*}$.

Now consider a time varying system $\dot{y}=X(t, y)$ with fixed point $y^{*}$. One faces a variety of difficulties when trying to adopt the program above to this situation: The linearized system $\dot{x}=D_{y} X\left(t, y^{*}\right) \cdot x=$ $A(t) x$ is time varying, hence the set of eigenvalues of $\{A(t), t \in \mathbb{R}\}$ may not describe the stability behavior of $A(t)$. Therefore, one has to use the Lyapunov exponents of $\{A(t), t \in \mathbb{R}\}$. But these exponents may not be regular, i.e. they are only defined as lim sup's, see e.g. Hahn (1967). Furthermore, the corresponding subspaces will now depend on time, which makes computations quite difficult. Finally, it is not obvious how to construct (time varying) invariant manifolds that reflect the stability behavior of the linear system in the behavior of the nonlinear system around $y^{*}$. For these (and other) reasons, the precise stability picture of $\dot{y}=X(t, y)$ at $y^{*}$ is extremely difficult to obtain.

Key words exd phrases. Lyapunov spectrum, Floquet spectrum, stability radii, stable manifolds.

Partially supported by DFG grant no Co 124/12-1 and by ONR grant no. N00014-93-1-0868 
Note however that if $A(t)$ is periodic, then stability analysis is feasible, e.g. via Floquet theory, Lyapunov transformations, or Poincaré maps.

An idea that yields a variety of stability results for time varying systems is to model the time dependency of $X(t, y)$ itself as a dynamical system. This can be done either for individual systems, i.e. one considers a flow $\{\psi(t), t \in \mathbb{R}\}$ on some state space, and assumes that the system is of the form $\dot{y}=X(\psi(t), y)$. Under certain conditions on $\{\psi(t), t \in \mathbb{R}\}$ one then obtains stability results for the time varying system, see e.g. Johnson and Nerurkar $(1992 \mathrm{a}, \mathrm{b})$. The other approach is to consider a family of time varying systems $\dot{y}=X(t, y)=X_{0}(y)+\sum_{i=1}^{m} u_{i}(t) X_{i}(y)$ where $u(t)=$ $\left(u_{i}(t), i=1 \ldots m\right)$ can be any measurable time varying function with values in some set $U \subset \mathbb{R}^{m}$. In this case, the set of time varying perturbations $u \in U$ is rich enough to model any (bounded) measurable time varying vector field, and one can define an associated (topological) flow on $U$. As a consequence, the Lyapunov exponents, their corresponding subspaces, and stable manifolds can be studied. This is the approach taken in this paper. In particular, we reduce the study of time varying, measurable vector fields to the study of a family of periodic systems, for which a detailed stability analysis is available.

In Section 2. we give a brief overview of constant and periodic linear systems in order to explain the essential features of stability analysis via Lyapunov exponents. Section 3. deals with time varying systems. We show which of the essentials can be retained for this class of systems. Finally, Section 4. is devoted to the stability study of nonlinear, time varying systems around fixed points. This is done via linearization, and the results of Section 3 . are utilized by means of a stable manifold theorem.

\section{REVIEW OF CONSTANT AND PERIODIC LIN- EAR SYSTEMS}

The analysis of constant coefficient, linear systems

$$
\dot{x}=A x \text { in } \mathbf{R}^{d}
$$

where $A \in g \ell(d, \mathbf{R})$, the $d \times d$ matrices with coefficients in $\mathbb{R}$, is a paradigm for many approaches in linear and nonlinear systems theory. Once a problem is reduced to one of the form (1), i.e. to a problem of linear algebra, it is considered 'solved'. Let us recall briefly, with respect to stability analysis, what can be said about the constant coefficient linear system.

The system (1) is exponentially stable iff it is globally asymptotically stable iff it admits a strict quadratic Lyapunov function iff all the eigenvalues of $A$ are in the left half of the complex plane. The system is (marginally) stable iff it admits a quadratic Lyapunov function iff all eigenvalues of $A$ have nonpositive real part and purely imaginary eigenvalues are geometrically simple. Actually, the eigenvalues and their associated (generalized) eigenspaces contain additional information: Let $\lambda_{1}<\lambda_{2}<\cdots<\lambda_{k}, k \leq d$, be the different real parts of the eigenvalues of $A$, with associated sums of generalized eigenspaces $E_{1} \ldots E_{k}$ of dimension $d_{1} \ldots d_{k}$. Let $e_{i j}, i=1 \ldots k, j=1 \ldots d_{i}$ be a basis of $\mathbb{R}^{d}$ with $e_{i j} \in E_{i}$ and consider an initial value $x_{0}=\sum_{i, j} \alpha_{i j} e_{i j}$. Define $i\left(x_{0}\right)=\max \left\{i, \alpha_{i j} \neq 0\right.$ for some $\left.j \in 1 \ldots d_{i}\right)$. Then the solution $\varphi\left(t, x_{0}\right)=e^{A t} x_{0}$ of $(1)$ behaves like $e^{\lambda_{i\left(x_{0}\right)^{t}}}$ for $t \rightarrow \infty$. In particular, we have $x_{0} \in E_{i}$ iff $\lim _{t \rightarrow \pm \infty} \frac{1}{t} \log \left|\varphi\left(t, x_{0}\right)\right|=\lambda_{i}$. Hence for $\lambda_{i}<0$ the system is exponentially stable from initial values $x_{0} \in E_{i}$, and exponentially unstable from $x_{0} \in E_{j}$ with $\lambda_{j}>0$. Consequently, $E^{s}=\bigoplus_{\lambda_{i}<0} E_{i}$ is the (exponentially) stable subspace of (1), the exponentially unstable subspace is $E^{u}=\bigoplus_{\lambda_{i}>0} E_{i}$, and $E_{i}$ for $\lambda_{i}=0$ is the center subspace $E^{c}$. Stabilization of the linear system (1) then amounts to changing the dynamics in $E^{u} \oplus E^{c}$. Furthermore, recall that a linear vector field $A \in g \ell(d, \mathbb{R})$ is structurally stable in $g \ell(d, \mathbb{R})$ iff $A$ is hyperbolic (has no eigenvalues with zero real part). This means that stability features of $A$ are preserved under small variation of its coefficients.

Turning for a moment to nonlinear systems, consider the differential equation

$$
\dot{x}=X_{0}(x) \text { in } \mathbb{R}^{d},
$$

where, for simplicity, $X_{0}$ is a $C^{\infty}$ vector field. Assume that $X_{0}$ has an isolated fixed point $x^{*} \in \mathbb{R}^{d}$, i.e. $X_{0}\left(x^{*}\right)=0$, and let without loss of generality $x^{*}=0$. The local behavior of the nonlinear system (2) around $x^{*}$ is described by the linearized system

$$
\dot{z}=A z \text { in } \mathbb{R}^{d}, A=D_{x} X_{0}\left(x^{*}\right) .
$$

There exist local $C^{\infty}$ manifolds $W^{*}, W^{u}, W^{c}$ at $x^{*}$ such that $\operatorname{dim} W^{s}=\operatorname{dim} E^{s}, \operatorname{dim} W^{u}=\operatorname{dim} E^{u}, \operatorname{dim} W^{c}=$ $\operatorname{dim} E^{c}$, and on $W^{s}$ and $W^{u}$ the system (2) has the same (exponential) stability behavior as that of the linear system (3) on $E^{s}$, and $E^{u}$ respectively. Furthermore, the invariant manifolds are tangential to the corresponding subspaces at the fixed point $x^{*}$. If $x^{*}$ is hyperbolic, then the local flow of (2) is equivalent to that of (3) (i.e. orbits are transformed into orbits via a homeomorphism), and the system (2) is locally structurally stable at $x^{*}$ under small perturbations within all $C^{r}, r \geq 1$ vector fields. 
Ideally, one would like to be able to analyze time varying systems in the same way as time invariant systems. While this is not possible in general, the program does go through for periodic systems

(4)

$$
\dot{x}=A(t) x \quad \text { in } \quad \mathbb{R}^{d}, A(T+t)=A(t) \text { for all } t \in \mathbb{R} \text {. }
$$

This can be seen in different ways, and we will present three approaches. Floquet theory implies that the fundamental matrix $\Phi(t)$ of (4) can be written in the form $\Phi(t)=P(t) e^{\Lambda t}$, where $P(t)$ is a $T$-periodic matrix function, and $\Lambda$ is a constant matrix containing the Floquet exponents in its diagonal. As in the time invariant case, $\Lambda$ contains the exponential stability information of the system, together with the stable, unstable, and center subspaces $E^{s}(0), E^{u}(0), E^{c}(0)$ for the initial time $t=0$. Then $E^{s}(t)=\Phi(t) E^{s}(0)=$ $P(t) E^{s}(0)$ changes $T$-periodically (although $\Phi(t)$ need not be periodic), and this is the stable subspace for time $t$. Analogously, $E^{u}(0)$ and $E^{c}(0)$ are transported via $\Phi(t)$ to the time periodic subspaces $E^{u}(t)$ and $E^{c}(t)$. Again, hyperbolic periodic linear systems are structurally stable within this class of systems.

Lyapunov transformations transform periodic linear systems (4) into time-invariant linear systems of type (1). This transformation is given by the solution of a linear, periodic matrix differential equation that preserves the exponential growth rates (hence the exponential stability behavior) and that maps the invariant subspaces of (4) into those of (1), and vice versa. It may be viewed as a $T$-periodic coordinate transformation. Compare e.g. Gantmacher (1959) for details on Lapunov transformations.

Poincaré maps can be utilized for the analysis of (4) in the following way: Define $w=\frac{2 \pi}{T}$ and consider the augmented system

(5) $\dot{\theta}=w$

$$
\dot{x}=A(\theta) x \text { in } \mathbb{R}^{d}
$$

on $\mathbb{S}^{1}$, the one-dimensional sphere, parametrized by the angle $[0,2 \pi)$.

Consider the global cross section $\Gamma=\left\{(x, 0), x \in \mathbb{R}^{d}\right.$, $\left.0 \in S^{1}\right\}$ and define the Poincare map $P: \Gamma \rightarrow \Gamma$ by $P(x, 0)=(\varphi(T, x), 0)$, where $\varphi$ is the solution of $(4)$, i.e. $P^{n}(x, 0)=\Phi(n T) x$, with $\Phi$ the fundamental matrix of (4). The discrete time system $\left\{P^{n}, n \in \mathbb{Z}\right\}$ is time invariant. The eigenvalues $\mu_{1} \ldots \mu_{d}$ of $P$ are related to the Floquet exponents $\lambda_{1} \ldots \lambda_{d}$ of (4) by $\lambda_{i}=\frac{1}{T} \log \left|\mu_{i}\right|$, and the invariant subspaces $E^{u}, E^{u}, E^{c}$ of $P$ generate the corresponding subspaces of (4) via $E^{\prime}(t)=\Phi(t) E^{s}$, $E^{u}(t)=\Phi(t) E^{u}, E^{c}(t)=\Phi(t) E^{c}$.
Turning again to nonlinear systems, assume that (2) has an isolated periodic solution $\varphi(t, x)$ with period $T>0$. Linearization of (2) around this periodic orbit leads to

$$
\dot{z}=A(t) z \quad \text { in } \quad \mathbb{R}^{d}, A(t)=D_{x} X_{0}(\varphi(t, x)) .
$$

The system (6) can be analyzed by the techniques described above, which leads to results for (2) locally around the periodic orbit $\gamma=\{\varphi(t, x), t \in[0, T)\}$ e.g. in the following way: Pick $p \in \gamma$, construct a local transveral cross section $\Gamma_{p}$ at $p$, and consider the Poincare map $P_{p}: \Gamma_{p} \rightarrow \Gamma_{p}$ of (2). Linearization of this map leads to eigenvalues and invariant subspaces as above. These invariant subspaces correspond to the stable, unstable and center manifolds $W_{p}^{s}, W_{p}^{u}, W_{p}^{c}$ of $P_{p}$. The flow $\Phi(t)$ of (2) maps these manifolds in $\Gamma_{p}$ into those of the periodic orbit $\gamma$. These invariant manifolds are tangential to the corresponding (time-varying) invariant subspaces of (6), displaying the same exponential growth behavior. Local equivalence of the flows (Grobman/Hartman Theorem) and local structural stability follow in the same way. We refer the reader to Wiggins (1988) for an account of the theory.

General time varying systems require a different approach to identify exponential growth rates, associated invariant subspaces, and corresponding invariant manifolds. The next section presents the linear theory, and Section 4. contains our results on nonlinear systems.

\section{TIME VARYING LINEAR SYSTEMS}

For general time varying linear systems, the approaches outlined in the previous section do not work: The real parts of the eigenvalues of $A(t), t \in \mathbf{R}$ do not necessarily determine the system's stability behavior, Lyapunov transformations exist only for so-called regular systems (compare again Gantmacher (1959)), and there are no obvious choices for transversal cross sections and Poincaré maps along trajectories. Therefore, a different approach has to be developed. In this section, we present our results in the following set-up.

Consider the family of linear, time varying systems

$$
\dot{x}=A(t) x=A_{0} x+\sum_{i=1}^{m} u_{i}(t) A_{i} x \text { in } \mathbb{R}^{d}
$$

where $\left(u_{i}\right)_{i=1 \ldots m}=u \in U=\{u: \mathbb{R} \rightarrow U$, measurable $\}$ and $U \subset \mathbb{R}^{m}$ is assumed to be convex, compact, with $0 \in$ int $U$, the interior of $U$. We call $U$ the perturbation range of the system (1). In order to be able to treat all bounded, measurable matrix functions $A(t)$, we introduce a range parameter $\rho \geq 0$ and define 
$U^{\rho}=\rho \cdot U=\left\{y \in \mathbb{R}^{m}\right.$, there exists $x \in U$ with $\left.y=\rho x\right\}$. Then $U^{\bullet}$ denotes the corresponding function space. Note that for all bounded, measurable matrix functions $A(t), t \in \mathbb{R}$ there are $A_{0} \ldots A_{m}, U \subset \mathbb{R}^{m}$ for some $m \leq d^{2}$, and $\rho \geq 0$ such that $A(t)=A_{0}+\Sigma u_{i}(t) A_{i}$ with $u \in \mathcal{U}^{\rho}$.

Exponential stability of the system (1) is measured by its Lyapunov exponents: Denote by $\varphi(t, x, u)$ the solution of (1) with initial value $x \in \mathbb{R}^{d}$ and perturbation $u \in U$. We define

(2)

$$
\begin{aligned}
& \lambda(u, x)=\limsup _{t \rightarrow \infty} \frac{1}{t} \log |\varphi(t, x, u)|,(u, x) \in U \times \mathbb{R}^{d}, \\
& x \neq 0 \text {. }
\end{aligned}
$$

For $u \equiv$ constant, the Lyapunov exponent $\lambda(u, x)$ is one of the real parts of the eigenvalues of $A(u)$; in fact $\lambda(u, x) \leq \lambda_{i}$ iff $x \in \bigoplus_{j=1}^{i} E_{j}$ (with notations from Section 2.). For $u(t)$ periodic $\lambda(u, x)$ is one of the Floquet exponents of $A(u(t))$. For general $u \in U$ the system (1) is exponentially stable for all initial values $x \in \mathbb{R}^{d}, x \neq$ 0 , if $\max _{x \neq 0} \lambda(u, x)<0$. Furthermore, for every $u \in U$ there are at most $d$ different Lyapunov exponents of $A(u(t))$. We will see later on that one also obtains generalized notions for $A(u(t))$ corresponding to the (generalised) eigenspaces.

In general it is difficult to analyze the Lyapunov exponents directly via the definition in (2). Instead, one separates the system (1) into an angular and a radial part. Since linear systems are homogeneous of order 1, it snffices to consider the angular part on the projective space $\mathbf{P}^{d-1}$ in $\mathbf{R}^{d}$. The projected system satiofies the differential equation

$$
\dot{s}=h(u, s)=h_{0}(s)+\sum_{i=1}^{m} u_{i}(t) h_{i}(s) \text { in } \mathbb{P}^{d-1}
$$

with $h_{j}(s)=\left(A_{j}-s^{T} A_{j} s \cdot I d\right) s, j=0 \ldots m$ where ${ }^{T}$ denotes transpose and $I d$ is the $d \times d$ identity matrix. Solving the corresponding (decoupled) linear equation for the radial part yields

$$
\begin{aligned}
& \lambda(u, s)=\limsup _{t \rightarrow \infty} \frac{1}{t} \int_{0}^{t} q(u(\tau), s(\tau)) d \tau, \\
& (u, s) \in U \times P^{d-1}
\end{aligned}
$$

where $q(u, s)=q_{0}(s)+\sum u_{i} q_{i}(s), q_{j}(s)=s^{T} A_{j} s$ for $j=0 \ldots m$. Since $\lambda(u, s)=\lambda(u, \alpha s)$ for all $\alpha \in \mathbf{R}$, $\alpha \neq 0,(4)$ describes all Lyapunov exponents of $A(u(t))$ for any initial value $x \neq 0$. In order to simplify our exposition, we will assume the following nondegeneracy condition for the projected system (3):

(H) $\operatorname{dim} \mathcal{L} A\{h(u, s), u \in U\}(s)=d-1$ for all $s \in \mathbb{P}^{d-1}$, where $\mathcal{L} A$ denotes the Lie algebra generated by the vector fields $\{h(u, s), u \in U\}$. Note again that for all bounded, measurable matrix functions $A(t), t \in \mathbf{R}$ we can find $A_{j}, j=0 \ldots m, U \subset \mathbb{R}^{m}, \rho \geq 0$ such that $A(t)=A_{0}+\Sigma u_{i}(t) A_{i}$ with $u \in \mathcal{U}^{*}$ and (H) is satisfied for $U^{p}$.

Define the Lyapunov spectrum of the system (1) for $\mathcal{U}^{\rho}$ as

$$
\Sigma_{L_{y}}(\rho)=\left\{\lambda(u, s), u \in \mathcal{U}^{p}, s \in \mathbb{P}^{d-1}\right\}
$$

A direct analysis of the Lyapunov spectrum is difficult, since the exponents $\lambda(u, s)$ need not depend continuously on $u$ nor on s. (Compare, however, Arnold and Nguyen (1994) for some continuity properties of stochastic Lyapunov exponents.) Therefore, we estimate $\Sigma_{L y}(\rho)$ from above using the so-called Morse spectrum, and from below via the Floquet spectrum. A comparison of these two spectra will yield the desired results.

The theory of the Morse spectrum is developed in Colonius and Kliemann (1995a) for general nonlinear systems, and the corresponding results for time varying linear systems are presented in Colonius and Kliemann (1995b). Since the results of this paper can be formulated without direct recourse to the Morse spectrum, we refer the reader to the two papers mentioned above for background material and proofs.

\subsection{THE FLOQUET SPECTRUM}

Spectra, being defined via time averages for $t \rightarrow$ $\pm \infty$, are defined on the limit sets of trajectories. In order to describe the Floquet spectrum, i.e. Lyapunov exponents over periodic orbits in the nonlinear equation, we first identify those sets on the projective space $\mathbb{P}^{d-1}$, where periodic orbits of the projected system (3) can be found.

1. Definition. A set $D \subset \mathbb{P}^{d-1}$ is called a control set of the system (3), if (i) $D \subset \mathrm{ClO}^{+}(x)$ for all $x \in D$, where $O^{+}(x)=\left\{y \in \mathbb{P}^{d-1}\right.$, there exist $u \in U$ and $t \geq 0$ with $\varphi(t, x, u)=y\}, \varphi$ denotes trajectories of (3), and " $c \varphi$ is the closure of a set, (ii) for all $x \in D$ there exists $u \in U$ such that $\varphi(t, x, u) \in D$ for all $t \geq 0$, and (iii) $D$ is maximal with properties (i) and (ii). Control sets with nonvoid interior are called main control sets.

It is shown in Colonius and Kliemann (1992) that the system (3) has $\ell \leq d$ main control sets $D_{1} \ldots D_{\ell}$, which are linearly ordered by the relation

$$
D_{k} \prec D_{j} \text { if there is } x \in D_{i} \text { with } \mathcal{O}^{+}(x) \cap D_{j} \neq 0 \text {. }
$$


Over the main control sets of (3) on $\mathbb{P}^{d-1}$ we define the Floquet spectrum of (1) as

(5)

$$
\Sigma_{F \ell}\left(D_{i}, \rho\right)=\left\{\lambda(u, s),(u, s) \in \mathcal{U}^{\rho} \times \mathbb{P}^{d-1}, \varphi(t, s, u)\right.
$$

is a periodic or constant solution of $(3)$ in int $\left.D_{i}\right\}$

$$
\Sigma_{F \ell}(\rho)=\bigcup_{i=1}^{\ell} \Sigma_{F \ell}\left(D_{i}, \rho\right) \text {. }
$$

Note that each $\lambda \in \Sigma_{F \ell}(\rho)$ is a Floquet exponent of some periodic system of the form (1). The following result was proved in Colonius and Kliemann (1995b).

2. Theorem. Consider the linear, time varying system ( 1 ) and assume (H). Then for each $i=1, \ldots \ell$ we have that $c \ell \Sigma_{F \ell}\left(D_{i}, \rho\right)$ is a bounded interval. Furthermore, if $D_{i} \prec D_{j}$ then $\Sigma_{F \ell}\left(D_{i}, \rho\right) \leq \Sigma_{F \ell}\left(D_{j}, \rho\right)$ in the sense that $\kappa_{i}^{*}(\rho) \leq \kappa_{j}^{*}(\rho)$ and $\kappa_{i}(\rho) \leq \kappa_{j}(\rho)$, where $\kappa_{i}^{*}(\rho)=\inf \Sigma_{F \ell}\left(D_{i}, \rho\right)$ and $\kappa_{i}(\rho)=\sup \Sigma_{F \ell}\left(D_{i}, \rho\right)$.

Of course, it holds that $\Sigma_{F \ell}(\rho) \subset \Sigma_{L_{y}}(\rho)$. In the next section we will study the question under which conditions the two spectra agree.

\subsection{THE LYAPUNOV SPECTRUM}

The Floquet spectral intervals $\Sigma_{F \ell}(D, \rho)$ increase with increasing $\rho \geq 0$. We introduce the following condition, which allows us to study the monotonicity of the Floquet spectrum (in $\rho$ ) in more detail.

(I) The system (3) has the $\rho-\rho^{\prime}$ inner pair property, if for $\rho<\rho^{\prime}$ it holds that for all trajectories $\varphi\left(t, x_{0}, u\right)$ in $\mathbb{P}^{d-1}$ with $u \in \mathcal{U}^{\rho}$ there exists a time $T \geq 0$ such that $\varphi\left(T, x_{0}, u\right) \in$ int $\mathcal{O}^{+, \rho^{\prime}}\left(x_{0}\right)$, where $\mathcal{O}^{+, \rho^{\prime}}\left(x_{0}\right)$ is the forward orbit of $x_{0}$ using controls in $\mathcal{U}^{p}$.

A detailed discussion of the inner pair property can be found e.g. in Colonius and Kliemann (1994). Under assumption (I) we obtain the following result.

3. Theorem. Consider the linear, time varying system (1) and assume (H) and (I).

(i) For each $i=1 \ldots k$ the map $\rho \mapsto D_{i}(\rho)$ is increasing, left continuous, and has at most $d$ discontinuity points.

(ii) For each $i=1 \ldots k$ the map $\rho \mapsto \Sigma_{F \ell}\left(D_{i}, \rho\right)$ is increasing, left continuous, and has at most countably many discontinuity points. It is dominated from above and below by a right continuous function, which agrees with the spectral map at its continuity points, in particular, $\rho=0$ is a continuity point.

(iii) At the continuity points $\rho$ of the spectral map in (ii) we have (a) $\kappa_{i}^{*}(\rho)<\kappa_{j}^{*}(\rho), \kappa_{i}(\rho)<\kappa_{j}(\rho)$ with $\kappa^{*}$ and $\kappa$ the infima and suprema of the Floquet spectral intervals (see Theorem 2);

(b) $\Sigma_{L_{y}}(\rho)=c \ell \Sigma_{F \ell}(\rho)$ (compare (5)).

(iv) $\min \Sigma_{L_{y}}(\rho)$ and $\max \Sigma_{L_{y}}(\rho)$ depend continuously on $\rho$.

Theorem 3. was proved in Colonius and Kliemann (1995b). As a consequence, we obtain the main result of this paper.

4. Theorem. Let $A(t)$ be a bounded, measurable matrix function and consider the linear, time varying system $\dot{x}=A(t) x$ in $\mathbb{R}^{d}$ with Lyapunov exponents $\lambda_{j}, j=$ $1 \ldots k$. Then for every $j$ and every $\varepsilon>0$ there exist a periodic (piecewise constant) matrix function $B(t)$ and a Floquet exponent $\mu_{j}$ of $B(t)$ such that $\left|\lambda_{j}-\mu_{j}\right|<\varepsilon$.

Proof. Choose $A_{0}, A_{i}$ and $u_{i}(t), i=1 \ldots m$ such that $A(t)=A_{0}+\sum_{i=1}^{m} u_{i}(t) A_{i}$. This is always possible by e.g. letting $m=d^{2}$. Denote $\rho:=$ ess sup $\|u(t)\|$, the essential supremum of $\|u(t)\|$, and set $U^{p}=\left\{v \in \mathbb{R}^{m},\|v\| \leq\right.$ $\rho\}$. One can always choose this set-up such that Assumptions (H) and (I) are satisfied, e.g. such that the projected system (3) has only one main control set on $\mathbb{P}^{d-1}$ for all $\rho>0$. This holds e.g. for $m=d^{2}$.

Note that $\rho$ as defined above need to be a continuity point of the spectral map in Theorem 3. (ii). But for any $\delta>0$ there exists $\rho^{\prime}$ with $\rho^{\prime}>\rho,\left|\rho-\rho^{\prime}\right|<\delta$ and $\rho^{\prime}$ is a continuity point. Let $\lambda$ be a Lyapunov exponent of $A(t)$, then by Theorem 3 . (iii) there exists for $\varepsilon>0$ a periodic $v^{0} \in \mathcal{U}^{\rho^{\prime}}$ such that a Floquet exponent $\mu_{0}$ of $B_{0}(t)=A_{0}+\Sigma v_{i}^{0}(t) A_{i}$ satisfies $\left|\lambda-\mu_{0}\right|<\frac{t}{2}$. A standard argument for periodic differential equations shows that a periodic, piecewise constant $B_{1}(t)$ with $v^{\prime} \in \mathcal{U}^{\rho^{\prime}}$ can be found such that a Floquet exponent $\mu_{1}$ of $B_{1}(t)$ satisfies $\left|\mu_{0}-\mu_{1}\right|<\frac{c}{2}$. Now set $B(t)=B_{1}(t)$.

This proof also shows that $B(t)$ can be chosen in such a way that its range is close to the range of $A(t)$, i.e. set $\sigma=$ ess $\sup \|A(t)\|$, then for all $\delta>0$ there exists a suitable $B(t)$ with ess sup $\|B(t)\|<\sigma+\delta$.

The Approximation Theorem 4. is not constructive in the way it stands now: The approximating periodic system $B(t)$ may have a large period, and it is not clear how to construct $B(t)$ from a given measurable matrix function $A(t)$. It is clear, however, that choosing $A_{0}, A_{i}$ and $u_{i}(t)$ for $i=1 \ldots m$ judiciously will result in smaller control sets and smaller spectral intervals which therefore approximate better the Lyapunov spectrum of a given system $A(t)$.

Before we turn to a variety of system theoretic implications of the results above, we discuss briefly the 
concept of 'eigenspaces' for time varying systems of the form (1). It is clear that the eigenspaces of $A(t)$ for $t \in \mathbb{R}$ do not have meaning for the stability behavior of the system, as explained for constant and time periodic systems in Section 2. However, we have the following decomposition result which clarifies the role of the main control sets.

5. Theorem. Consider the linear, time varying system (1) and assume (H) and (I). Let $\rho$ be a continuity point of the spectral map in Theorem 3. (ii), and let $D_{i}(\rho)$ be the corresponding main control sets.

(a) For each $p \in$ int $D_{i}(\rho)$ there exists a periodic $u(t) \in U^{p}$ such that $p \in E_{j}^{p}(u)$ for some $j=1 \ldots d$, where $E_{j}^{p}(u)$ is an eigenspace of the fundamental matrix at time $T$, the period of $u(t)$. Equivalently, $E_{j}^{\rho}(u)$ is an eigenspace of the associated Poincare map, see Section 2.

(b) In general, define for $u \in \mathcal{U}^{\rho}: E_{i}^{\rho}(u)=\{x \in$ $\mathbb{R}^{d}, x \neq 0$ implies $\varphi(t, x, u) \in c l D_{i}(\rho)$ for $t \in$ $\mathbb{R}$ \}. Then the $E_{i}^{\rho}(u)$ are subspaces of dimension independent of $u \in \mathcal{U}^{p}$, invariant under the flow of the system (1), and $\mathbb{R}^{d}=E_{1}^{\rho}(u) \oplus$ $\cdots \oplus E_{\ell(\rho)}^{\rho}(u)$ where $\ell_{(\rho)}$ is again the number of main control sets for the projected system (3) with range $U^{p}$. The dimension $\operatorname{dim} E_{i}^{p}(u)$ is the sum of the dimensions of the (generalized) eigenspaces of $A_{0}$ that are contained in $c\left(D_{i}(p)\right.$.

Theorem 5. shows that for general linear time varying systems one can still define 'eigenspaces' $E_{i}^{\rho}(u)$, but they are, in general, a coarser decomposition of $\mathbb{R}^{d}$ than eigenspaces for constant or time periodic systems. In analogy to Section 2. we define for $u \in U^{p}$

(6)

$$
\begin{aligned}
& E_{\rho}^{\prime}(u)=\oplus E_{i}^{\prime}(u), \kappa_{i}(\rho)<0, \text { stable subspace, } \\
& E_{\rho}^{u}(u)=\oplus E_{i}^{\prime}(u), \kappa_{i}^{\circ}(\rho)>0, \text { unstable subspace, } \\
& E_{\rho}^{c}(u)=\oplus E_{i}^{\prime}(u), 0 \in c \Sigma_{F \ell}\left(D_{i}, \rho\right), \text { center subspace. }
\end{aligned}
$$

These subspaces describe the uniform stability behavior of the family of time varying systems (1), as explained in the next section.

\subsection{STABILITY OF LINEAR, TIME VARYING SYS- TEMS}

Exponential stability of time varying linear systems is described by the system's Lyapunov exponents. The theory developed in the previous sections leads to the following results. According to Theorem 3. the spectral intervals are increasing and left continuous in the perturbation size $\rho$. This leads to a schematic picture:

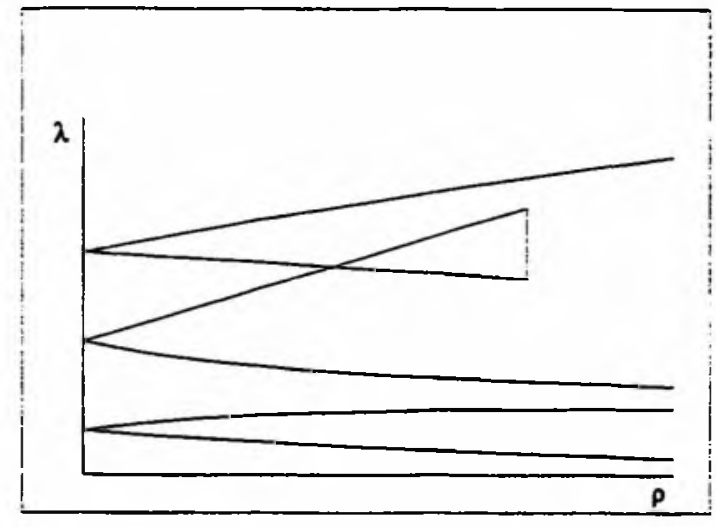

Figure 1. Spectral intervals depending on the perturbation size $\rho$.

For $\rho=0$, the spectrum consists of the real parts of the eigenvalues of $A_{0}$. For increasing $\rho$, the main control sets develop around the corresponding (sums of generalized) eigenspaces of $A_{0}$, and the spectral intervals increase. They are ordered by the order of the main control sets, but they may overlap (see Figure 1.). With increasing $\rho$, control sets may merge and then the corresponding spectral intervals unite. (In general, it is not possible to compute the Lyapunov exponents, and hence the spectral intervals, analytically. See Grüne (1995) for the numerics of spectral intervals and explicit examples.) As a consequence, we can describe the exponential stability behavior of the family (1) of time varying systems at a continuity point of the spectral map in Theorem 3. (ii). (For the study of discontinuity points we refer the reader to Colonius and Kliemann (1995b, 1993)).

Let $\rho$ be a continuity point of the spectral maps $\rho \mapsto c \ell \Sigma_{F \ell}\left(D_{i}, \rho\right), i=1 \ldots \ell(\rho)$. The system (1) with initial value $x_{0} \in \mathbb{R}^{d}$ is exponentially stable from $x_{0}$ if $x_{0} \in E_{\rho}^{z}(u)$. This means that (1) is stable from all $x_{0} \in \mathbb{R}^{d}, x_{0} \neq 0$, such that the trajectory $\varphi\left(t, x_{0}, u\right)$ of the projected system has an $\omega$-limit set on $\mathbb{P}^{d-1}$ which is contained in Ucl $D_{i}(\rho)$, where the union is taken over all main control sets with $\kappa_{i}(\rho)<0$. In particular, the family of systems $(1)$ is exponentially stable for all $u \in U^{\rho}$ and all $x_{0} \in \mathbb{R}^{d}$ iff $\max \Sigma_{L_{y}}(\rho)<0$. The results for instability are completely analogous using $E_{\rho}^{u}(u)$. Similar to the constant coefficient case, one can prove that if $\max \Sigma_{L_{y}}(\rho)>0$, then for an open an dense set of $u \in \mathcal{U}^{p}$ and $x_{0} \in \mathbb{R}^{d}$ the system response is unstable (see Colonius and Kliemann (1995b)). Furthermore, if the minimum of the largest spectral interval is positive, then for all $u \in U^{p}$ there exists a dense set of 
initial values $x_{0} \in \mathbb{R}^{d}$ such that the system response is unstable. For initial values in the center space $E_{\rho}^{c}(u)$ no general results can be obtained. For $x_{0} \in E_{\rho}^{c}(u)$ the individual Lyapunov exponent $\lambda\left(u, x_{0}\right)$ has to be computed. Again, $\lambda\left(u, x_{0}\right)<0$ means exponential instability, and $\lambda\left(u, x_{0}\right)=0$ means exponential growth or decay, or marginal stability.

An equivalent to structural stability (see Section 2. for constant coefficient and periodic systems) for timevarying systems (1) is obtained from Theorem 3 . in the following way: Assume that the system $\left(1^{p}\right)$ is hyperbolic in the sense that $E_{\rho}^{c}(u)=0$ for all $u \in U^{\rho}$, i.e. $0 \notin \Sigma_{L_{y}}(\rho)$.

6. Theorem. Consider the linear time varying system $(1)$ and assume (H) and (I). Let $\rho$ be a continuity point of the spectral maps. If the system $\left(1^{p}\right)$ is hyperbolic, then there exists a $\delta>0$ such that for all $\rho^{\prime} \in(\rho-$ $\delta, \rho+\delta)$ we have

(a) The system $\left(1^{\rho^{\prime}}\right)$ is hyperbolic,

(b) $\operatorname{dim} E_{\rho}^{s}(u)=\operatorname{dim} E_{\rho^{\prime}}^{s}(v)$ for all $u \in \mathcal{U}^{\rho}$ and $v \in \mathcal{U}^{\rho^{\prime}}$, compare (6).

Furthermore, for $\rho=0$ we obtain that the stability behavior of $A_{0}$ is preserved under small time varying perturbations in $\mathcal{U}^{p^{\prime}}, \rho^{\prime}<\delta$.

Proof.

(a) If the system $\left(1^{\rho}\right)$ is hyperbolic, then $\left(1^{\rho^{\prime}}\right)$ is hyperbolic for $\rho^{\prime} \leq \rho$ because the spectral intervals are increasing. For $\rho<\rho^{\prime}$ consider w.l.o.g. a spectral interval $\Sigma_{F \ell}\left(D_{i}, \rho\right)$ with $\kappa_{i}(\rho)<0$. Since $\rho$ is a continuity point of $\kappa_{i}(\rho)$, there exists $\rho>0$ such that $\kappa_{i}\left(\rho^{\prime}\right)<0$ for all $\rho^{\prime}<\rho+\delta$, which proves (a) for $\rho>0$. At $\rho=0, \kappa_{i}$ is dominated from above by a right continuous map $\alpha(\rho)$ with $\lim _{\rho \backslash 0} \alpha(\rho)=\kappa_{i}(0)$, the corresponding Lyapunov exponent of $A_{0}$. By right continuity of $\alpha$ we again obtain $\delta>0$ such that $\kappa_{i}\left(\rho^{\prime}\right)<0$ for all $\rho^{\prime}<\delta$, if $\kappa_{i}(0)<0$.

(b) At continuity points of the spectral maps, the control set maps $\rho \mapsto D_{i}(\rho)$ are continuous, see Theorem 3. Hence the number of control sets $\ell\left(\rho^{\prime}\right)$ is constant in a neighborhood of $\rho$. By Theorem 5. (b) the result follows for $\rho>0$. At $\rho=0$, the control set maps are again dominated by a right continuous map, see Colonius and Kliemann (1995b), and hence $\ell(\rho)$ is constant on $[0, \delta)$ for some $\delta>0$. Now the result follows as above.

Theorem 6., when applied to the maximal Lyapunov exponent

$$
\kappa(\rho)=\max \Sigma_{L_{y}}(\rho)=\sup \Sigma_{F \ell}(\rho)
$$

leads to the notions of robust stability and stability radii under time varying perturbations. In the present set-up, these concepts and their characterizations were developed in a series of papers by the authors, see Colonius et al. (1992) for a survey of the results. Here we only mention the basic definition and some immediate consequences.

7. Definition. Consider the constant coefficient matrix $A_{0} \in g \ell(d, \mathbb{R})$ and a family of perturbation ranges $\left\{U^{\rho}, \rho \geq 0\right\}$ leading to the time varying systems (1). Define the stability radius of $A_{0}$ with respect to $\left\{U^{P}\right.$, $\rho \geq 0\}$ by

$$
\begin{array}{r}
r\left(A_{0}\right)=\inf \left\{\rho \geq 0, \text { there exists } u \in U^{\rho}\right. \text { such } \\
\text { that } \dot{x}=\left(A_{0}+\sum_{i=1}^{m} u_{i}(t) A_{i}\right) x \\
\text { is not exponentially stable }\} .
\end{array}
$$

The following result is an immediate consequence of Theorem 6.

8. Corollary. (a) $r\left(A_{0}\right)=\inf \{\rho \geq 0, \kappa(\rho) \geq 0\}=$ $\sup \{\rho \geq 0, \kappa(\rho)<0\}$.

(b) $r\left(\bar{A}_{0}\right)>0$ iff $A_{0}$ is exponentially stable.

Similarly to Definition 7 . stability radii can be defined for time-varying matrix functions $A(t)$. Embed$\operatorname{ding} A(t)$ into a system of the form (1) (see the proof of Theorem 4.) leads to sufficient criteria of the form: $r(A(t))>0$ if $\kappa(\rho)<0$, where $\kappa(\rho)$ is defined as in (7) and $U^{\rho}$ is a range such that $A(t) \in\left\{A_{0}+\Sigma u_{i} A_{i}, u \in\right.$ $\left.U^{\rho}\right\}$. (The result follows directly from Theorem 6 . and the continuity of $\kappa(\rho)$, see Theorem 3. (iv).) The setup presented in this paper is, to the best of our knowledge, the only framework that allows the study of persistence of stability for arbitrary time varying systems $\dot{x}=A(t) x$ under general $L^{\infty}$ perturbations.

\section{TIME VARYING NONLINEAR SYSTEMS}

The nonlinear analogue of the linear, time varying system (3.1) is

$$
\dot{y}=X(t, y)=X_{0}(y)+\sum_{i=1}^{m} u_{i}(t) X_{i}(y) \text { in } \mathbb{R}^{d}
$$

with $u \in \mathcal{U}^{\rho}$ as in Section 3. Exponential convergence and divergence of the trajectories of $(1)$ is again measured by the Lyapunov exponents, now defined for the linearized flow of the system (1) on the tangent bundle, or, equivalently, for the projected flow on the projective bundle, compare (3.3) and (3.4). A stable manifold theorem (compare Section 2.) then describes how 
to utilize the results on the linearized system for the original nonlinear one. This program can be carried out in great generality, compare e.g. Colonius and Kliemann (1995d). Here we restrict ourselves to linearization around a fixed point, where the results of Section 3. apply directly. Note also that the state space of (1) could be a smooth manifold, resulting in only minor notational complications.

We assume for the rest of this section that the system (1) has an isolated fixed point $y^{*} \in \mathbb{R}^{d}$, i.e. $X_{0}\left(y^{*}\right)$ $=\cdots=X_{m}\left(y^{*}\right)=0$. Linearization around $y^{*}$ results in the system

$$
\dot{x}=A(t) x=A_{0} x+\sum_{i=1}^{m} u_{i}(t) A_{i} x \text { in } \mathbb{R}^{d},
$$

where $A_{j}=D_{y} X_{j}\left(y^{*}\right)$ for $j=0 \ldots m$, and $D_{y} X_{j}\left(y^{*}\right)$ denotes the Jacobian of the vector field $X_{j}$ at $y^{*}$. The perturbations $u \in \mathcal{U}^{p}$ are defined as before. Of course, (2) is a system of the type (3.1), and therefore all results described in the previous section hold for the linearized system at $y^{*}$.

As explained in Section 2., a stable manifold theorem allows us to transfer the linear situation to the nonlinear system, locally around $y^{*}$. A result on stable manifolds of (1) at $y^{*}$ is obtained as follows:

1. Theorem. Assume that the vector fields $X_{j}, j=$ $0 \ldots m$ are smooth, and that the linearized system (2) satisfies Assumptions (H) and (I) from Section 3. Let $\rho$ be a continuity point of the spectral maps in Theorem 3.3 (ii) and let $D_{1}(\rho) \ldots D_{r}(\rho)$ be the main control sets of the projected system (3.3) with $\kappa_{i}(\rho)<0, i=$ $1 \ldots$. . Then there exist topological manifolds $W_{p}^{s}(u)$ with dimension $\operatorname{dim} W_{p}^{s}(u)=\operatorname{dim} E_{p}^{s}(u)=\sum_{i=1}^{r} \operatorname{dim} E_{i}^{p}(u)$ (defined as in (3.6)) such that for all $y \in W_{\rho}^{s}(u)$ we have $\lim _{t \rightarrow \infty}\left|\varphi(t, y, u)-y^{*}\right|=0$, where $\varphi(t, y, 0)$ denotes the solutions of the nonlinear system (1).

The stable manifolds $W_{p}^{\prime}(u)$ are local objects at $y^{*}$, but they are uniform in the sense that there exists $\delta>0$ such that for all $u \in U$ the $W_{p}^{\prime}(u)$ are defined in the $\delta$-neighborhood $\left\{y \in R^{d},\left|y-y^{*}\right|<\delta\right\}$ of $y^{*}$. Furthermore, since the stable manifolds according to Theorem 1. are (only) topological manifolds, we do not have a notion of tangentiality of $E_{p}^{z}(u)$ and $W_{p}^{\prime}(u)$. But the subspaces and the manifolds are close at $y^{*}$ in the sense that their Lipschitz distance goes to 0 as they approach $y^{*}$, compare Colonius and Kliemann (1995d) for a precise formulation of this statement and for a proof of Theorem 1., which is based on an abstract stable manifold theorem in Bronstein and Chernii (1978).
Using Theorem 1., the spectral and stability results of Section 3. carry over to the nonlinear, time varying system (1) at the fixed point $y^{*}$. In particular, we obtain in a nonlinear analogue of Theorem 3.4., and Theorem 3.6. reads in its nonlinear version:

2. Corollary. Consider the nonlinear, time varying system (1) with isolated fixed point $y^{*}$. Assume (H) and (I) for the linearized system (2), and let $\rho$ be a continuity point of the spectral maps. If the fixed point $y^{*}$ of $\left(1^{p}\right)$ is hyperbolic (i.e. if the linearized system $\left(2^{\rho}\right)$ is hyperbolic) then there exists a $\delta>0$ such that for all $\rho^{\prime} \in(\rho-\delta, \rho+\delta)$ we have

(a) the fixed point $y^{*}$ of $\left(1^{\rho^{\prime}}\right)$ is hyperbolic,

(b) $\operatorname{dim} W_{\rho}^{s}(u)=\operatorname{dim} W_{\rho^{\prime}}^{s}(v)$ for all $u \in U^{p}$ and $v \in \mathcal{U}^{p^{\prime}}$.

Furthermore, for $\rho=0$ we obtain that the stability behavior of $\dot{y}=X_{0}(y)$ at $y^{*}$ is preserved under small time varying perturbations in $\mathcal{U}^{\rho^{\prime}}, \rho^{\prime}<\delta$.

Similarly, the stability and instability results in Section 3.3. carry over as local results at the fixed point $y^{*}$ of the nonlinear, time varying system (1). In particular we have (see Colonius and Kliemann (1995c))

3. Corollary. Let $y^{*}$ be an isolated fixed point of the system (1), and suppose that the linearized system satisfies Assumption (H).

(a) If $\kappa(\rho):=\sup _{u \in \mathcal{U} \cdot x \neq 0} \sup _{x \neq} \lambda(u, x)>0$, then the fixed point $y^{*}$ is unstable.

(b) If $\kappa(\rho)<0$, then $y^{*} \in$ int $W_{p}^{*}(u)$ for all $u \in \mathcal{U}^{p}$.

As in the linear case, Corollary 3. and the continuity of $\kappa(\rho)$ in $\rho$ allow us to define a nonlinear stability radius of the system $\dot{y}=X_{0}(y)$ at the fixed point $y^{*}$ as

$$
\begin{aligned}
& r_{n \ell}\left(y^{*}\right)=\inf \left\{\rho \geq 0, \text { there exits } u \in U^{\rho} \text { such that } y^{*}\right. \\
& \text { is not exponentially stable for } \\
& \left.\qquad \dot{y}=X_{0}(y)+\sum_{i=1}^{m} u_{i}(t) X_{i}(y)\right\} .
\end{aligned}
$$

The following result summarizes some results on the nonlinear stability radius $r_{n \ell}$. The proof and further properties of $r_{n \ell}$ can be found in Colonius and Kliemann (1995c).

4. Corollary. Under the assumptions of Corollary 3. we obtain:

(a) $r_{n \ell}\left(y^{*}\right) \geq r\left(A_{0}\right)$ with $A_{0}=D_{y} X_{0}\left(y^{*}\right)$ and $r\left(A_{0}\right)$ as in Definition 3.7.

(b) $r_{n \ell}\left(y^{*}\right)>0$ iff $y^{*}$ is exponentially stable for $\dot{y}=X_{0}(y)$. 
Finally, we would like to point out two problems that should be addressed in order to complete the theory of time varying systems as presented in this paper. First of all, note that the center subspace as defined in (3.6) can be rather large, and will, in general, contain points from which the system response is exponentially stable, see Colonius and Kliemann (1993). Hence a more detailed analysis of this subspace is necessary in order to obtain a result on center manifolds for the nonlinear, time varying system (1). Secondly, our stable manifold theorem does not imply the equivalence of the linear flow and the nonlinear flow around $y^{*}$ (Grobman/Hartman type theorem). However, such a result would clarify the possible behavior of the nonlinear, time varying system (1) locally around the fixed point $y^{*}$. Both results together could lead to a precise bifurcation theory for nonlinear, time varying systems.

\section{REFERENCES}

Arnold, L., and Nguyen, D.C., 1994 "Generic properties of Lyapunov exponents," Random and Computational Dynamics, Vol. 2, pp. 335-345.

Bronstein, I.U., and V.F. Chernii, V.F., 1978, "Linear extensions satisfying Perron's condition $I$," Differential Equations,' Vol. 14, pp. 1234-1243.

Colonius, F., Kliemann, W., and Krull, S., 1992, "Stability and stabilization of linear uncertain systems - a Lyapunov exponents approach," Report no. 372 of the Schwerpunktprogramm der Deutschen Forschungsgemeinschaft 'Anwendungsbezogene Optimierung und Steuerung', Augsburg.

Colonius, F., and Kliemann, W., 1992, "Linear control semigroups acting on projective space," J. Dynamics Diff. Equations, Vol 5, pp. 495-528.

Colonius, F., and Kliemann, W., 1993, "Asymptotic null-controllability of bilinear systems," Proceedings of the Woorkshop 'Geometry in Nonlinear Control' Warsaw, June 1993.

Colonius, F., and Kliemann, W., 1994, "Limit behavior and genericity for nonlinear control systems," J. Differential Equations, Vol 109, pp. 8-41.

Colonius F., and Kliemann, W., 1995a, "The Morse spectrum of linear flows on vector bundles," to appear in: Trans. AMS.

Colonius, F., and Kliemann, W., 1995b, "The Lyapunov spectrum of families of time varying matrices," to appear in Trans. AMS.

Colonius, F., and Kliemann, W., 1995c, "A stability radius for nonlinear differential equations subject to time varying perturbations," Proceedings of IFAC 'Symposium on Nonlinear Control Systems Design', Tahoe City, CA, June 1995.
Colonius, F., and Kliemann, W., 1995d, "The dynamics of control systems," in preparation.

Gantmacher, F.R., 1959, "The Theory of Matrices," Chelsea.

Grüne, L., 1995, "Numerical stabilization of bilinear control systems," submitted.

Hahn, W., 1967, "Stability of Motion," SpringerVerlag, New York.

Johnson, R., and Nerurkar, M., 1992a, "On null controllability of linear systems with recurrent coefficients and constrained control," J. Differential Equations.

Johnson, R., and Nerurkar, M., 1992b, "Stabilization and linear regulator problem for linear nonautonomous control processes," preprint.

Wiggins, S., 1988, "Global Bifurcations and Chaos : Analytical Methods," Springer-Verlag, New York. 